\title{
Crisis económica y financiera en los negocios que radicaron quiebra con el Capítulo 11 de Reorganización en Puerto Rico
}

\section{Financial and economic crisis of businesses in Puerto Rico that filed bankruptcy under Chapter 11 of Reorganization}

\section{Crise Econômica e Financeira nos negócios que estabeleceram a quebra com o Capítulo 11 de Reorganização em Puerto Rico}

\author{
Emmanuel Guzmán Rodríguez \\ Carlos F. Rosa Vázquez
}

\section{RESUMEN}

El estudio examina el problema de las quiebras en Puerto Rico, y la posibilidad de que estas puedan afectar negativamente a los negocios y a su entorno social y comercial. Los análisis están fundamentados en datos secundarios obtenidos con una muestra probabilística de 126 negocios seleccionada con un $95 \%$ de nivel de confiabilidad y un margen de error de $5 \%$. Se encontró que la crisis en este sector empresarial es ocasionada por un manejo no apropiado de los recursos financieros, en conjunto con la situación económica que ha resultado en una disminución del flujo monetario del país y la necesidad de financiamiento continuo con una economía en recesión/ depresión, pudiera provocar que la empresa radique un proceso de reorganización en la corte de quiebras. Este proceso se puede utilizar como una estrategia gerencial para mejorar el manejo de sus activos y pasivos corrientes en un plazo de tiempo establecido por la corte. Los resultados también muestran que algunos de los negocios pequeños, medianos y de dueños propios en Puerto Rico han experimentado el efecto de las quiebras en cadena, cuyas repercusiones tienen un efecto social y en otras entidades comerciales.

Palabras clave: crisis financiera, crisis económica, reducción del flujo de efectivo, poder adquisitivo, eficiencia gerencial, insuficiencia de capital, deterioro de inventario.

\section{ABSTRACT}

The study examinesthe problemof bankruptciesinPuerto Rico,andthe possibility thatthesemay adversely affect the businessand its socialand commercial environment. The analysesare basedon secondary data obtained with a probability sample of 126 businessesselectedwith a $95 \%$ confidence leveland a margin oferror of $5 \%$. It was found thatthe crisis in thebusiness sector were causedby impropermanagement of financialresources, together with the economic situationthat has resulted ina

Bachillerato en Contabilidad Pública, Maestría en Contribuciones, Maestría en Contabilidad, Universidad del Turabo, Puerto Rico. Correspondencia con el autor: emanguz@hotmail. com.

** DBA, MBA, ASQ-CQIA, CED, Catedrático asociado, Universidad del Turabo, Puerto Rico. Correspondencia con el autor: carlosrosa_692002@yahoo.com; crosa31@suagm.edu. 
Crisis económica y financiera en los negocios que radicaron quiebra con el Capítulo 11 de Reorganización en Puerto Rico / Guzmán y Vázquez

decrease incash flowin the countryand the need forcontinued fundingwith an economy in recession/ depression, couldcause thecompany to file for bankruptcy under chapter 11 of reorganization. Thisprocess can be usedas a managementstrategy to improvethe management of theirassetsand liabilitieswithin a period of time set bythe court. The results also showthat some of thesmall, mediumandsole proprietorships owners inPuerto Ricohave experiencedthe effect ofbankruptcies; and the repercussions have asocial and commercial impact.

Keywords: financial crisis, economic crisis, reduced cash flow, purchasing power, managerial efficiency, insufficient capital, inventory impairment.

\section{RESUMO}

O estudo analisa o problema de quebras em Puerto Rico, e a possibilidade de que estas possam afetar adversamente aos negócios e seu ambiente social e comercial. As análises estão fundamentadas em dados secundários obtidos com uma amostra probabilística de 126 negócios selecionados com um nível de confiança de $95 \%$ e uma margem de erro do 5\%. Encontrou-se que a crise neste sector empresarial é causada por uma gestão não apropriada dos recursos financeiros, em conjunto com a situação econômica que resultou numa diminuição do fluxo monetário do país e a necessidade de financiamento continuo com uma economia em recessão / depressão, o que pode provocar que a empresa estabeleça um processo de reorganizaçáo na Corte de Quebras. Este processo se pode utilizar como uma estratégia de gestão para melhorar a administração dos seus ativos e passivos correntes dentro de um período de tempo fixado pelo tribunal. Os resultados mostram também dâo conta que alguns dos negócios pequenos, médios e de proprietários de Puerto Rico, têm experimentado o efeito das quebras em cadeia, onde as repercussóes têm um impacto social e outras entidades comerciais.

Palavras-chave: crise financeira, crise econômica, redução de fluxos de efetivo, poder aquisitivo, eficiência de gestáo, insuficiência de capital, deterioro de inventário.

\section{Planteamiento del problema}

La crisis financiera y económica en Puerto Rico ha impactado de manera negativa a los negocios, provocando en ellos la necesidad de considerar el remedio legal de radicar quiebra, con el fin de reorganizarse y utilizar esta estrategia legal para intentar alcanzar sus objetivos empresariales y de negocio. Es de conocimiento general que las empresas en Puerto Rico están vulnerables a cambios económicos como la inflación, incremento en los gravámenes, la recesión/depresión (Lobato, 2012) y la imposición de nuevos impuestos; por lo tanto, cualquier cambio que incremente el desembolso de los comerciantes augura su insolvencia y la necesidad de radicar quiebra con el capítulo 11 para reorganizar sus deudas y sobrevivir por varios ańos. Por esta razón se prepondera que el alza de las quiebras radicadas en Puerto Rico con el capítulo 11 de Reorganización esté relacionada con la recesión/depresión económica del país. Este estudio considera hallar respuesta al problema planteado. 


\section{Justificación del problema}

El problema de las quiebras del capítulo 11 necesita prioridad en ser solucionado porque su anuncio de bancarrota se percibe que tendrá efectos negativos en su entorno social. Más preocupante cuando la posible razón de las quiebras sea por una crisis económica y financiera del negocio. La economía de Puerto Rico ha experimentado una recesión constante desde el año 2006. Factores macroeconómicos en disminución pueden ser indicadores de una reducción de efectivo disponible en la sociedad y no un problema causado por la gerencia. Las empresas privadas sostienen que ante la condición económica del país, el contratar más personal les crearía problemas de liquidez. Su opinión se justifica con el incremento de sus gastos corrientes, les reduciría su margen de ganancias y resultaría un nuevo ciclo en la disminución de empleados contratados, aumento en la carga económica del gobierno, y la radicación de quiebra por el consumidor al ser despedido y no poder cumplir con sus responsabilidades económicas. Un efecto en cadena con forma de bola de nieve.

\section{Importancia del estudio}

Esta investigación es importante porque estudia y considera este fenómeno como un problema de interés social, económico y legal. Las quiebras radicadas en Puerto Rico pueden utilizarse como un indicador económico, porque tienen un conglomerado de efectos negativos como: despidos, reducción de jornadas de trabajo, incremento en el precio de los servicios/productos disponibles, y otros como la disminución en pagos contributivos al vender/proveer menos servicios a sus consumidores. Los negocios son una fuente de ingresos tanto para la sociedad como para el gobierno; por esto, se realiza una investigación exploratoria en Puerto Rico para conocer quiénes se afectan por los negocios que radicaron quiebra y si las crisis económica y financiera son las causas principales de las quiebras registradas con el capítulo 11 de reorganización.

Las quiebras tienen la capacidad de incrementar la crisis económica y financiera de su entorno comercial y social. Si las estadísticas de quiebras en Puerto Rico continúan causarán la disminución en el poder adquisitivo de las personas y resultará en un nuevo ciclo de quiebras de negocios y personas. El ciclo del flujo de efectivo doméstico en Puerto Rico sostiene e incrementa la calidad de vida de su sociedad. Esto sucede si el flujo de efectivo del país no tiene escapes por distintos métodos, como el pago de instrumentos financieros al exterior y transferencias de ingresos devengados a bancos de otros países. Los pagos y las transferencias sustanciales de dinero al exterior disminuyen la cantidad 
Crisis económica y financiera en los negocios que radicaron quiebra con el Capítulo 11 de Reorganización en Puerto Rico / Guzmán y Vázquez

de efectivo disponible en Puerto Rico y por consecuencia empobrecen a la sociedad del país.

\section{Marco conceptual y contextual}

\section{El rol de la crisis financiera y crisis económica}

Los distintos instrumentos financieros como bonos, acciones y préstamos comerciales son métodos utilizados para incrementar los recursos monetarios de una entidad a cambio de pagar una determinada cantidad pre acordada o esperada. La crisis financiera comienza cuando la entidad no genera suficiente flujo de efectivo para pagar sus deudas y gastos según vencen. Esto ocasiona un daño en el historial crediticio, los préstamos son otorgados con mayores intereses e incluso pueden ser denegados, el valor de los bonos disminuye por la percepción de que la entidad no podrá pagarlos en su vencimiento, y las acciones se afectan por la percepción negativa de los inversionistas. La crisis económica ocurre cuando la entidad no tiene flujo de efectivo, con la desvalorización en los bonos y en las acciones se obtiene menos dinero que la cantidad a pagar, y/o los ingresos devengados no son suficientes para cumplir con todas sus deudas y gastos según vencen. Teniendo en cuenta que los efectos causados por la crisis financiera aumentan el desembolso de efectivo y agotan los recursos monetarios de la entidad. Por consecuencia, la crisis financiera puede causar la crisis económica y la combinación de estas resulta en la necesidad de radicar quiebra al no tener recursos monetarios.

South, Swenson \& Saunders (2004) mencionan que las únicas opciones para reducir una crisis financiera es aumentar los ingresos, reducir los costos, o vender los activos. Es razonable pensar que estos factores tienen la influencia de reducir la crisis financiar al incrementar el flujo de efectivo y aumentar el margen de ingresos netos devengados. Los fideicomisos asignados por la Corte Federal de Quiebra Distrito de Puerto Rico reorganizan la condición económica y financiera del negocio al incrementar el flujo de efectivo, reducir gastos, posponer el pago de deudas, y vender los activos del negocio. Dresner \& Windle (2009) discuten que el estrés financiero tiene influencia en el comportamiento de los precios de las empresas. Incluso mencionan la existencia de una relación positiva entre las empresas que radican quiebra y la disminución del precio en sus productos y/o servicios. Estrategia económica que intenta incrementar el flujo de efectivo al aumentar la demanda mediante la reducción del precio de un producto o servicio.

Borenstein \& Rose (2003) mencionan que la reducción de la demanda, costos sostenidos o incrementos, y la disponibilidad de sustitutos de un producto o servicio tienen el potencial 
para crear la crisis financiera; por consecuencia pudiera concluir con una radicación de quiebra con el capítulo 11 de Reorganización. Los estudios de South, Swenson \& Saunders (2004), Dresner \& Windle (2009), Sturm \& Winters (2010) y Borenstein \& Rose (2003) tienen concordancia, que los factores dominantes para causar la crisis financiera son la disminución de los ingresos con un incremento de gastos sostenido.

Existen indicadores de crisis financiera como el pago de intereses sobre el valor en el mercado (Stevenson, 2000). Fujiwara (2008) menciona los siguientes indicadores que causan el fracaso de diversos negocios: un desempeño pobre, recesión/depresión económica en la industria, excesiva competencia, choques extrínsecos, una gerencia floja, conflictos internos, falta de eficiencia gerencial, déficits económicos prolongados, insuficiente capital en funcionamiento, desastres accidentales, el deterioro de inventario, y la inversión excesiva en facilidades y equipo. Estos indicadores pueden crear una crisis financiera e influir en la radicación de quiebra, sin crear un efecto de quiebras en cadena en su industria o en la sociedad (Fujiwara, 2008).

\section{Metodología}

Se realizó un estudio exploratorio tomando en consideración que los factores de crisis económica y financiera son la causa principal de que los negocios en Puerto Rico necesiten radicar quiebra con el capítulo 11 de reorganización. Es un estudio cuantitativo y cualitativo basado en información secundaria que intenta describir, explicar, y correlacionar las variables exploratorias de crisis económica y financiera. Se consideraron varias hipótesis alternas con el objetivo de proponer relaciones y poner a prueba la relación entre las variables y los negocios que radicaron quiebra con el capítulo 11 de reorganización en Puerto Rico. Se propone que los resultados de las hipótesis deben ayudar a explicar qué, cómo y por qué las variables afectan a los negocios en Puerto Rico.

\section{Muestra seleccionada}

Se utilizó el programa de STATS para la selección de los elementos muestrales. Los criterios establecidos han sido de un nivel de confiabilidad de 95\% y un nivel de error estandarizado $(Z)$ máximo de 5\% para estimar una muestra del 10\% que represente el $90 \%$ de la población. Con una población de 1.448 el resultado ha sido una muestra de 126 negocios. La muestra de 126 empresas se ha distribuido anualmente al dividir las cantidades anuales de 125, 119, 110, 80, 96, 155, 158, 177, 214 y 214 entre la población de 1.448 negocios, y luego multiplicada por la muestra de 126 para obtener la cantidad de negocios a investigar por cada año [(Quiebras anuales/Población $)^{*}$ muestra]. 
Crisis económica y financiera en los negocios que radicaron quiebra con el Capítulo 11 de Reorganización en Puerto Rico / Guzmán y Vázquez

\section{Descripción de la población}

El total del sujeto de estudio es de 1.448 negocios que radicaron quiebra con el capítulo 11 de reorganización entre el 2004 hasta diciembre 2013; y que ya tengan resolución jurídica, que hayan sido desestimados o que todavía estén en el proceso de quiebra (United States Courts, Bankruptcy Statistics). La población seleccionada para el estudio son pequeños negocios, medianos negocios y personas con sus propios negocios que voluntariamente radicaron quiebra con el capítulo 11 de reorganización y que tienen su dirección física y realizan negocios en Puerto Rico.

\section{Procedimientos}

La muestra de 126 negocios a investigar será obtenida de los Archivos de la Corte Federal de Quiebras Distrito de Puerto Rico. Se ha creado una cuenta para registrarse en su sistema y pagar las copias digitales que tienen un costo de 10 centavos por página, más el costo del índice de cada caso legal de quiebra. La muestra anual ha sido seleccionada según su fecha, comenzando por las primeras del año hasta completar la cantidad identificada de cada año.

\section{Hipótesis Descriptivas:}

Hd1: Los negocios de Puerto Rico que radicaron quiebra con el capítulo 11 de reorganización tenían indicadores de necesidad de radicar quiebra con el capítulo 11 de reorganización.

Hd2: Los negocios de Puerto Rico que radicaron quiebra con el capítulo 11 de reorganización lo hicieron para incrementar su flujo de efectivo.

\section{Hipótesis Explicativas:}

He1: La crisis financiera causó la necesidad de que los negocios de Puerto Rico radicaran quiebra con el capítulo 11 de reorganización.

He2: La disminución en el flujo de efectivo (crisis económica) causó la necesidad de que los negocios de Puerto Rico radicaran quiebra con el capítulo 11 de reorganización.

\section{Resultados}

Se pudo establecer que la crisis económica más la crisis financiera tienen el potencial para crear la necesidad de que los negocios radiquen quiebra con el capítulo 11 de 
reorganización. A cada término se le puede asignar una serie de indicadores que ayuden a predecir la necesidad de un negocio de tener que radicar quiebra. Los siguientes factores han sido identificados como parte de la crisis económica: disminución en el flujo de ingresos con un incremento o balance en las cuentas por pagar y balances sustanciales en cuentas por cobrar. Los siguientes están relacionados con la crisis financiera: balances de deudas no aseguradas mayores al total de activos, inversión excesiva en activos no corrientes y el pago de gastos operacionales con préstamos y tarjetas de crédito.

El diccionario de la Real Academia Espańola asigna tres definiciones a la palabra déficit: cuando las deudas sobrepasan el total de activos, cantidad a financiar necesaria para cubrir gastos, y la falta de recursos monetarios para cumplir con las responsabilidades. Se pudo identificar que de una muestra de 126 negocios durante los años 2004 hasta el 2013, el 89,68\% (113/126) de los negocios radicaron quiebra por déficit, ya que tenían la necesidad de incrementar sus préstamos para cubrir gastos y no tenían suficiente flujo de efectivo para cumplir con sus deudas y obligaciones según vencían. Cuando se extrapolan estos resultados a la población de 1.448 empresas se puede estimar que $1.298\left(1.448^{*} 0,8969\right)$ radicaron quiebra porque no obtenían más financiamiento para cubrir sus gastos y no tenían suficiente flujo de efectivo para cumplir con sus responsabilidades según vencían. Los datos de la segunda hipótesis descriptiva y explicativa demuestran que el 100\% (126) de los negocios radicaron quiebra con el propósito de incrementar su flujo de efectivo. Aun cuando el 10,32\% (13/126) de los negocios radicaron quiebra por estrategias legales, económicas y financieras, como descongelar cuentas bancarias, para cambiar los montos y términos de sus deudas, y para mantener el mismo estilo de vida por los dueños de los negocios, tenían el propósito de incrementar su flujo de efectivo. Este resultado, al ser extrapolado a la población de 1.448 negocios que radicaron quiebra en Puerto Rico, se puede concluir que el 100\% radicó quiebra con el propósito de incrementar su flujo de efectivo y $149\left(1.448^{*} 0,1032\right)$ negocios radicaron quiebra como estrategia legal, económica o financiera para incrementar su flujo de efectivo.

La primera hipótesis explicativa prueba que los negocios que radicaron quiebra pueden tener crisis financiera pero no tener crisis económica. Este tipo de empresa tiene flujo de efectivo pero se le hace difícil aumentar su financiamiento. Entonces radica quiebra como estrategia para incrementar su flujo de efectivo, disminuir sus gastos a corto plazo, conservar sus activos y adquirir financiamiento por Orden de la Corte Federal de Quiebra Distrito de Puerto Rico. En el 100\% de los negocios que radicaron quiebra se encontraron indicadores de inestabilidad económica y financiera. Los indicadores económicos y financieros son reflejados en la lista de acreedores, el listado de propiedades personales y en el Estado de Situación reportado. 
Crisis económica y financiera en los negocios que radicaron quiebra con el Capítulo 11 de Reorganización en Puerto Rico / Guzmán y Vázquez

De una muestra de 126 negocios estudiados, dos (1,58\%) negocios presentaron varios indicadores del efecto de las quiebras en cadena. Estas dos empresas fueron parte de este fenómeno porque una persona era el mismo dueño (a) de múltiples negocios, utiliza el dinero de los negocios para la compra de propiedades personales, combina las finanzas de los negocios, y toma decisiones negligentes como financiar las operaciones del negocio con tarjetas de crédito. Se considera que otros dos negocios (1,58\%) fueron parte del efecto de las quiebras en cadena y tuvieron la necesidad de radicar quiebra al no recibir los ingresos de sus cuentas por cobrar. Basado en la información presentada se estima que $46\left(1.448^{*} 3,17 \%\right)$ de 1.448 negocios que radicaron quiebra fueron parte o causaron el efecto de quiebras en cadena. Este resultado confirma que distintos sectores económicos han sido afectados por los negocios acogidos a la quiebra del capítulo 11 de reorganización en Puerto Rico.

\section{Conclusiones}

Se puede concluir que la existencia de los factores crisis económica y financiera son suficientes para crear la necesidad de que un negocio radique quiebra. La ecuación para determinar si un negocio tiene la necesidad de radicar quiebra sería: Necesidad de Quiebra con el Capítulo 11 es igual a la Crisis Económica más la Crisis Financiera (Necesidad Quiebra Capítulo 11= CE + CF). Se suman porque es el conjunto de los indicadores de estos dos factores los que predicen al 100\% la necesidad de quiebra. Se pudo encontrar que los indicadores a utilizar se dividen por factor. La crisis económica tiene los indicadores de disminución en el flujo de ingresos con un incremento o balance en las cuentas por pagar y balances sustanciales en cuentas por cobrar. Los indicadores del factor crisis financiera son: balances de deudas no aseguradas mayores al total de activos, inversión excesiva en activos no corrientes, y el pago de gastos operacionales con préstamos y tarjetas de crédito. Una limitación del modelo para predecir quiebra con una probabilidad de $100 \%$ es que depende de la voluntad de la gerencia para comenzar el proceso, o que sus acreedores les obliguen legalmente a una quiebra involuntaria.

Aun cuando este modelo pueda predecir la necesidad de quiebra, no será de utilidad si el país tiene una disminución en el flujo monetario y los negocios tienen un financiamiento continuo con una economía en recesión/depresión. Basado en este ambiente económico se podría utilizar este modelo para intentar predecir si un negocio que radicará quiebra tiene el potencial de crear el efecto de quiebras en cadenas, o si algún negocio será afectado por las quiebras radicadas.

Según la información recopilada algunos de los negocios pequeños, medianos y de dueños propios en Puerto Rico han experimentado el efecto de las quiebras en cadena, 
e incluso se pudo encontrar que dos negocios causaron el efecto de quiebras en cadena. Puerto Rico tiene una economía peor que estancada (recesión, más inflación, más aumento en la pérdida de empleos) porque está en una continua depresión, con una inflación continua y una pérdida de empleos en incremento. Basado en este modelo de predecir la necesidad de radicar quiebra, se puede predecir que los negocios de Puerto Rico están predeterminados a radicar quiebra con el capítulo 11.

\section{Recomendaciones}

Es importante y prioritario renovar e innovar las teorías económicas que no tienen validez en la economía de Puerto Rico. Los principios de las teorías económicas son muy buenos, pero existen variables que en Puerto Rico causan que estas no puedan utilizarse como la dependencia y la limitación de los productos importados al país. Se ha mencionado que todo negocio que dependa sustancialmente de otros negocios tiene la predisposición de radicar quiebra. Además de desarrollar nuevas teorías económicas se deben desarrollar medidas financieras que limiten las cantidades prestadas o que las instituciones bancarias monitoreen su uso para evitar que se utilice el dinero para pagar gastos de operaciones. Todo negocio que pague sus operaciones con financiamiento tiene la predisposición de radicar quiebra y causar el efecto de las quiebras en cadena. Se recomienda aplicar el modelo de predecir quiebra a todos los negocios de Puerto Rico para intentar identificar si estos tienen la capacidad de crear el efecto en cadena.

También es importante realizar un estudio que tome en cuenta a las personas que se afectan en el proceso del efecto en cadena, porque un negocio puede afectar a otro pero en cada uno se pueden tener cientos de empleados que serán afectados y posiblemente terminen en quiebra para aumentar las pérdidas de múltiples acreedores en distintas industrias. El peor escenario sería que una empresa con poder económico en Puerto Rico radique quiebra y miles de empleados queden sin empleos en una economía que no puede sostener o incrementar la fuerza trabajadora. Esto resultaría en el efecto de quiebras en cadena en grandes proporciones con innumerables efectos económicos y financieros.

\section{Bibliografía}

Accounting Terms (2010). Dictionary of accounting terms. (5 $5^{\text {th }}$ Ed.). Hauppauge, NY: Shim J. K. \& Siegel J. G.

Álvarez, M. (2013). Quiebras y préstamos en Puerto Rico: Una mirada exploratoria a su relación. Ecos de Economía: Universidad de EAFIT. Escuela de Economía 
Crisis económica y financiera en los negocios que radicaron quiebra con el Capítulo 11 de Reorganización en Puerto Rico / Guzmán y Vázquez

y Finanzas. ISSN. 1657-4206, No. 36, enero-junio 2013, pp. 127-146. Medellín, Colombia.

American Airlines se Declara en Quiebra (2011, noviembre 29). Primera Hora.

Benmelech, E. \& Bergman, N. (2011). Bankruptcy and the Collateral Channel. The Journal of Finance, Vol. 66, No. 2, april.

Borenstein, S. \& Rose, N. L. (2003). The impact of bankruptcy on airline service levels. American Economic Association Papers and Proceedings: Competition Policy in Network Industries, Vol. 93, No. 2, may.

Campbell M. \& Hernández F. D. (2010). Cross-border insolvencies: liquidation cases in the United Statess with extraterritorial effects within Argentina. Insolvency and Restructuring International, Vol. 4, No. 1

CIA (2010). Producto Interno Bruto por persona en Puerto Rico. Obtenido el 10 de abril de 2014, en https://www.cia.gov/library/publications/the-worldfactbook/geos/rq.html

Cintron, D. \& Wright, M. (2008). Bankruptcy costs, leverage and multiple secured creditors: the case of management buy-outs. Accounting and Business Research, Vol. 38, No. 1, pp. 71-89.

Doane, D. P. \& Seward, L. E. (2011). Applied Statistics in Business \& Economics. (3th Ed.). New York, NY: McGraw-Hill International Edition.

Finance and Investment Terms (2010). Dictionary of Finance and Investment Terms. ( $8^{\text {th }}$ Ed.). Hauppauge, NY: Downes J. \& Goodman J. E.

Fujiwara Y. (2008). Chain of firms' bankruptcy: a macroscopic study of link effect in a production network. Advances in Complex Systems, Vol. 11, No. 5 (2008) 703-717 (c) World Scientific Publishing Company.

General Motors (2013/11/15). About General Motors. Obtenido de http://careers. gm.com/about-gm.html

Haensly, P. J.; Theis, J. \& Swanson Z. (2003). Reassessment of Contagion and Competitive Intra-industry Effects of Bankruptcy Announcements. Quarterly Journal of Business \& Economics, Vol. 40, Nos. 3 and 4.

Johnson, M. A. \& Mamum, A. (2012). The failure of Lehman Brothers and its impact on other financial institutions. Applied Financial Economics, 2012, 22, 375385. doi: 10.1080/09603107.2011.613762.

Kryzkowski, B. (2009, july). Automotive Crash Course: Domino effect of GM, Chrysler bankruptcies. Wilson Web, Qual Prog 42, No. 7. Obtenido en www.qualityprogress.com. 
Lanciano, R. J.; Farrell, F. M.; Reed, E. A. \& Debote, P. (2013). Regulation: Business Law \& Professional responsibility. Lambers: CPA Review.

Lobato, V. M. (2012). El sistema financiero y la recesión económica en Puerto Rico: una visión panorámica. UPR, FAE. Obtenido el 23 de abril de 2014, en cicia.uprpp.edu/publicaciones/Papers/.../Lobato-Sist_Fro_y_Recesion.pdf.

Lucci, J. P. (2003). Enron-the bankruptcy Heard around the World and the international richochet of Sarbanes-Oxley. Albany Law Review, Vol. 67.

Maroto, M. (2012, september). The scarring effects of bankruptcy: cumulative disadvantage across credit and labor markets. SocialForces 91(1) 99-130, september, doi: 10.1093/sf/sos095.

Miller, H. R. (2007). Chapter 11 in Transition - From Boom to Bust and Into the Future. American Bankruptcy Law Journal, Vol. 81, pág. 375-403.

Milton C., Taylor (1954). Puerto Rico: Recovery or Relapse? The American Journal of Economics and Sociology. Volume 14, April, 1955, Number 3.

Real Academia Española (2001). Diccionario de la Lengua española (22nd Ed.). Madrid, Spain: n/a.

Rivera, M. \& Álvarez, Y. (2013, mayo 7). Augura avalancha de quiebras por el Ivutazo. El Vocero.

Rosa V., C. F. (2010). Fundamentos finanzas mercantiles - un enfoque gerencial. Puerto Rico.

Sampieri, H. R.; Collado, F. C. \& Lucio, B. P. (2010). Metodología de la investigación (5 ${ }^{\text {ta }}$ Ed.). México: McGraw-Hill/Interamericana editores, S.A. de C.V.

Sieczka, P.; Sornette, D. \& Holyst, J. A. (2011). The Lehman Brothers effect and bankruptcy cascades. The European Physical Journal B, 82, 257-269, doi: 10.1140/epjb/e2011-10757-2.

South, B. G.; Swenson, J. E. A. \& Saunders, G. A. (2004). Current issues affecting utility bankruptcies. The Journal of Structured and Project Finance, pp. 3441 Winter.

Stevenson, S. (2000). Contagion effects and intra-industry information flows: the example of Olympian \& York. Journal of Property Research, 17(2), 133-145.

Sturm, R. R. \& Winters, D. B. (2010). The Effect of Bankruptcy on U.S. Air Fares. Research in Applied Economics, Vol. 2, No. 2: E2. ISSN: 1948-5433.

Surendranath, R. J. \& Madura, J. (2010). The long-run performance of firms emerging from chapter 11 bankruptcy. Applied Financial Economics, 2010, 20, 11451161. ISSN 1466-4305 online. DOI: 10.1080/09603101003761895. 
Crisis económica y financiera en los negocios que radicaron quiebra con el Capítulo 11 de Reorganización en Puerto Rico / Guzmán y Vázquez

United States Courts (2013). Bankruptcy Statistics. Obtenido de http://www.uscourts. gov/Statistics/BankruptcyStatistics.aspx.

Wong, M. K. (2003). Bankruptcy as a Risk Management Tool: Economic and Social implications. Review of Business, Fall 2003.

\section{Cómo citar este artículo:}

Guzmán, E. y Rosa, C. (2014). "Crisis económica y financiera en los negocios que radicaron quiebra con el Capítulo 11 de Reorganización en Puerto Rico”, Oikos № 37, 93 - 104, Escuela de Administración y Economía, Universidad Católica Silva Henríquez (UCSH), Santiago de Chile.

[http://ediciones.ucsh.cl/revistas.php]

Fecha de recepción: 18 / 10 /2014

Fecha de aceptación: 05 / 12 / 2014 